\title{
Validation of Household Food Insecurity Access Scale - Sri Lanka Validation of HFIAS-SL
}

\author{
H.V.B.S Wijayatilaka ${ }^{1}$, Dulitha N. Fernando ${ }^{2}$
}

\begin{abstract}
Background

Until recently, Sri Lanka did not have a validated instrument with which to assess household food insecurity. The objective of this study was to adapt and validate the Household Food Insecurity Access Scale, which has been developed and used by the United States Agency for International Development's Food and Nutrition Technical Assistance project, in Sri Lanka.
\end{abstract}

\section{Methods}

This tool, Household Food Insecurity Access Scale, consists of nine questions which were adapted to the local setting with input and approval from both local content experts and target community members. The adapted tool was pretested and identified as the Household Food Insecurity Access Scale-Sri Lanka (HFIAS-SL) and was used to assess the household foodinsecurity. One hundred and fifty households from different socio-economic levels were purposively identified and included in the sample. Wealth index and household per capita income were assessed as indicators of socio-economic status. Food intake was assessed using a food group frequency questionnaire.

\section{Results}

Cronbach's Alpha was 0.879 (95\% CI: 0.847 - 0.906) and scale question response curves were parallel across the socio-economic strata of the households. The two extreme levels, 'food secure' and 'severely food insecure' households, showed a statistically significant dose-response relationship $(P<0.001)$ across wealth index and household per capita income strata. Daily consumption of food from animal sources and fruit had a statistically significant dose-response relationship $(P<0.001)$ with household food insecurity.

\section{Conclusion}

The Household Food Insecurity Access Scale-Sri Lanka is a valid tool to assess household food insecurity in Sri Lanka.

Key words : Validation, Food Insecurity, Sri Lanka, HFIAS

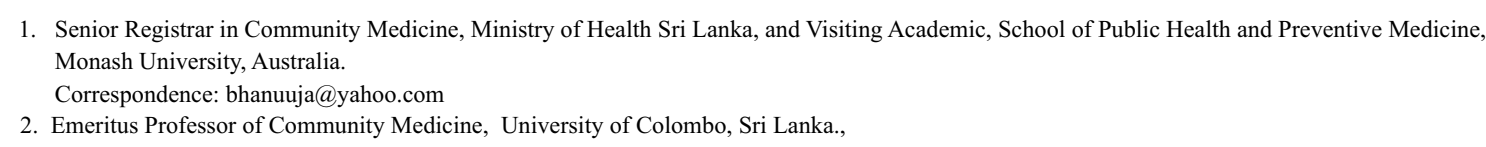




\section{Introduction}

Malnutrition is a multifaceted problem and its associated factors have been comprehensively illustrated in the UNICEF conceptual framework (1), where household food insecurity (HFI) is identified as an important underlying factor for malnutrition.

Food insecurity is a complex multidimen-sional concept and therefore, measuring it has been an ongoing challenge to researchers and practitioners (2). It has been assessed indirectly using energy balance sheets and child anthropometry. However, these approaches have not always been useful for guiding food insecurity, hence the need for the use of more direct experiential approaches (3). In response to this need, the Food and Nutrition Technical Assistance (FANTA) project of the United States Agency for International Development (USAID) has developed Household Food Insecurity Access Scale (HFIAS), a direct and user-friendly approach for measuring household food insecurity (2). A national level study conducted in Sri Lanka to assess complementary feeding practices has used this tool to assess HFI and recommended the validation of this tool in the Sri Lankan setting(4). Thus, in the absence of a validated instrument in Sri Lanka to assess HFI, this study aimed at validating the HFIAS as an instrument to assess HFI at the community level in Sri Lanka.

USAID defines household food security as, "when all people at all times have both physical and economic access to sufficient food to meet their dietary needs for a productive and healthy life" (5). Although there are several dimensions which reflect the household food insecurity including a household's access to enough food, nutritional adequacy and a household's ability to obtain it through normal channels (6), the measure presented in HFIAS, focuses only on the central dimension, "access to enough food", as perceived and reported by adult members of the household.

\section{Study design}

Validation is the process of determining whether a method is suitable for providing useful analytical measurement for a given purpose and context and is carried out by appraising judgmental validity and assessing the internal and external validity of the tool(7).

Judgmental validity assesses whether the conceptual definition has been translated appropriately into operational terms(8). Face validity, content validity and consensual validity are considered for judgmental validity. This HFIAS has been developed through a series of activities including field validation studies and consultative workshops by an expert group(2). Therefore, this scale was considered to have demonstrated a high level of judgmental validity. In addition, the tool was appraised by adapting it into the local setting, using the procedure recommended by Coates et al.(2).

\section{Internal validity}

Internal validity was assessed by evaluating the internal consistency of the questionnaire. Cronbach's alpha, which measures the extent to which the responses to each item in the scale correlates, was used. This method has been recommended and used in previous studies $(3,7)$.

\section{External validity}

Criterion validity is the most appropriate method to assess the external validity of an instrument when there is an agreed superior measure or 'gold standard', to measure the variable concerned(8). 
When such a measure is not available, a study instrument can be assessed against other known measures by assessing the convergent validity(8). As it was difficult to find a 'gold standard' to assess the household food insecurity, the external validity of HFIAS was evaluated in this study by assessing the convergent validity according to the approaches used and criteria recommended by Perez-Escamilla et al.(3) and Frongillo(7). The measures used for this purpose represented the household socio-economic status andconsumption of 'selected food groups'.

\section{Study setting}

The study unit was a household, defined as group of people who share food and lodging under one roof. Purposively selected households belonging to different socio-economic levels were identified from a Medical Officer of Health $(\mathrm{MOH})$ area. Such a sample was necessary for the assessment of convergent validity. Households with at least one child aged between 12 to 59 months and residents who had been in the study area for a period of more than one month were included.

\section{Selection of households}

Different sample sizes have been used for validation studies $(3,9)$. As suggested by Frangillo and Nanama, a purposive sample that could be manageable logistically with adequate coverage of the diversity of the experience of food insecurity was chosen(10).

Identification of households was done with the assistance of field level health staff, Public Health Midwives (PHMs) who had prior knowledge of the socio-economic levels of the households. Based on the available information on socio-economic background, households were chosen from the upper, middle and lower socio-economic strata. From each stratum, 50 households were selected, enabling the inclusion of 150 households.

\section{Method of data collection and key variables}

The tool, HFIAS was translated into Sinhala language and adapted to the study setting according to the procedure recommended(2). This adapted tool was pretested and identified as the Household Food Insecurity Access Scale-Sri Lanka (HFIAS-SL), which was used to assess HFI in this study. Furthermore, an interviewer administered questionnaire was used to collect information to determine other key variables: wealth index (WI), household per capita income (HPCI) and food intake (FI). WI and HPCI were used to represent the household socio-economic status. Mothers of children aged $12-59$ months responded to all questions in all households except one, where the grandmother was the caregiver. A high level of privacy and confidentiality was maintained especially during the data collection for HFIAS-SL, as it included sensitive questions.

\section{HFIAS-SL}

HFI was determined using the HFIAS-SL which included nine questions. Each question received one of the main two responses; "Yes" or 'No'. "Yes" responses were further subdivided into 'rarely', 'sometimes' or 'often'. 'No' indicated food security and other three responses (rarely, sometimes or often) to a particular question indicated the degree of food insecurity. Households with "No" responses to all nine questions or households with "No" responses to last eight questions and responded as "rarely" for the first question were included under category of "food secure households" while all other households were categorized as "food insecure households" (Table 1). Severity of those "food insecure households" was further categorized into three levels (mild, moderate and severe) by considering the combination of responses using the criteria stated by Coates et al(2), as illustrated in Table one. 
Table 1 Household food security/insecurity categorization scheme

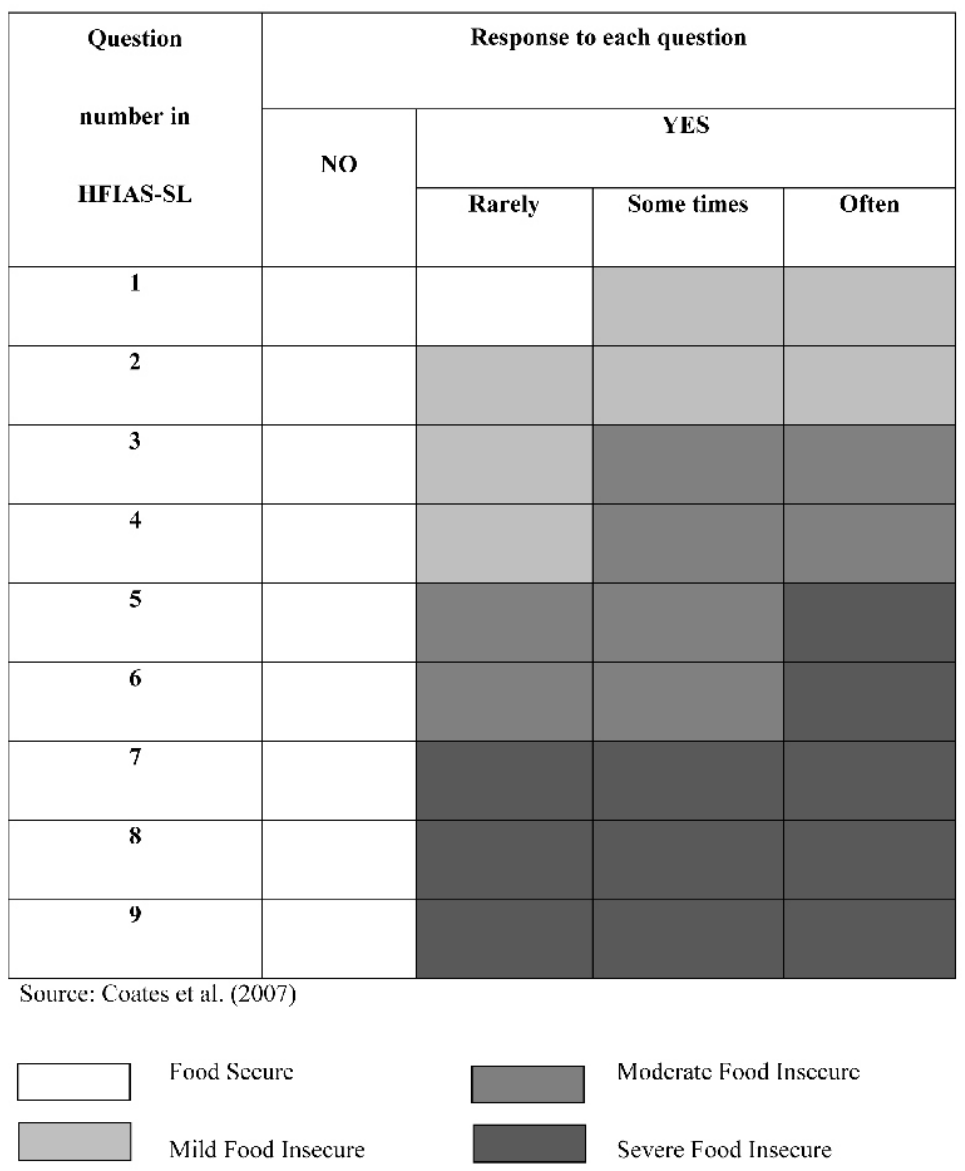

\section{Wealth index}

WI is a composite variable assessed using the availability of household assets and is not dependent on household income. The variables (assets) ${ }^{1}$ used by the Department of Census and Statistics, Sri Lanka in the Demographic and Health Survey 2006/2007 were used to assess the wealth index(11). Principal component analysis was performed to assign weights to each household asset and a wealth score for each household was obtained. The households were divided into four mutually-exclusive WI categories.

\section{Household per capita income}

Household per capita income (HPCI) was calculated by taking the total household income and number of household members into account. Households were categorized into four groups based on the quartiles of HPCI.

\section{Food intake}

Intake of different food groups was assessed using a 'food group frequency questionnaire'. Food groups were decided on the basis of their nutritional value and their cost. The questionnaire obtained information on the number of days during the previous week, during which different food groups were made available for consumption for the household members.

\section{Data analysis}

The analysis focused on: assessment of internal consistency, parallelism on question-response curves across socio-economic strata, doseresponse relationship between level of household food insecurity and socioeconomic strata, doseresponse relationship between levels of household food insecurity and consumption of 'selected food groups', and the correlation between household food insecurity and socioeconomic strata. 
Table 2 The distribution of the households by levels of HPCI, WI and HFI

\begin{tabular}{|c|c|c|c|}
\hline \multirow{2}{*}{ Variable } & \multicolumn{3}{|c|}{ Study sample $(\mathrm{n}=150)$} \\
\hline & No & $\%$ & Cumulative $\%$ \\
\hline \multicolumn{4}{|c|}{ Household Per Capita Income } \\
\hline Lowest quartile & 36 & 24.0 & 24.0 \\
\hline Lower medium quartile & 39 & 26.0 & 50.0 \\
\hline Upper medium quartile & 38 & 25.3 & 75.3 \\
\hline Highest quartile & 37 & 24.7 & 100.0 \\
\hline \multicolumn{4}{|l|}{ Wealth Index } \\
\hline Lowest & 57 & 38.0 & 38.0 \\
\hline Lower medium & 22 & 14.7 & 52.7 \\
\hline Upper medium & 23 & 15.3 & 68.0 \\
\hline Highest & 48 & 32.0 & 100.0 \\
\hline \multicolumn{4}{|l|}{ Household food insecurity } \\
\hline Food Secure & 42 & 28.0 & 28.0 \\
\hline Mild Food Insecure & 45 & 30.0 & 58.0 \\
\hline Moderately Food Insecure & 37 & 24.7 & 82.7 \\
\hline Severely Food Insecure & 26 & 17.3 & 100.0 \\
\hline
\end{tabular}

Ethical clearance for the study was obtained from the Ethics Review Committee of the Faculty of Medicine, University of Colombo.

\section{Results}

A total of 150 households was included and the response rate was $100 \%$. The distribution of the households by levels of HPCI, WI and HFI is presented in Table 2.Cronbach's Alpha for all nine questions was $0.879(95 \% \mathrm{CI} ; 0.847$ to
0.906). The value increased to 0.881 when question four of the scale was excluded and to 0.885 when question nine was excluded.

\section{Assessment for parallelism on question- response curves across socio-economic strata}

The percentages of 'yes' responses to each question in the scale were analysed to determine their association with the socio-economic strata of the households represented by WI and HPCI. 
Table 3 'Yes' responses to questions in the HFIAS-SL by wealth index

\begin{tabular}{|c|c|c|c|c|c|c|c|c|c|}
\hline \multirow{4}{*}{$\begin{array}{l}\text { Items } \\
\text { in the } \\
\text { HFIAS-SL }\end{array}$} & \multicolumn{8}{|c|}{ Wealth Index $(\mathrm{n}=150)$} & \multirow{4}{*}{$\begin{array}{l}\text { Test for } \\
\text { statistical } \\
\text { significance } \\
(\chi 2 \text { for linear } \\
\text { trend })\end{array}$} \\
\hline & \multirow{2}{*}{\multicolumn{2}{|c|}{$\begin{array}{l}\text { Lowest } \\
(n=57)\end{array}$}} & \multirow{2}{*}{\multicolumn{2}{|c|}{$\begin{array}{l}\text { Lower middle } \\
(\mathrm{n}=22)\end{array}$}} & \multirow{2}{*}{\multicolumn{2}{|c|}{$\begin{array}{l}\text { Upper middle } \\
(n=23)\end{array}$}} & \multirow{2}{*}{\multicolumn{2}{|c|}{$\begin{array}{l}\text { Highest } \\
(\mathrm{n}=48)\end{array}$}} & \\
\hline & & & & & & & & & \\
\hline & No & $\%$ & No & $\%$ & No & $\%$ & No & $\%$ & \\
\hline Q1 & 53 & 92.9 & 15 & 68.1 & 10 & 43.4 & 8 & 16.6 & $64.478 ; p<0.01$ \\
\hline Q2 & 57 & 100.0 & 20 & 90.9 & 15 & 65.2 & 16 & 33.3 & $59.708 ; \mathrm{p}<0.01$ \\
\hline Q3 & 46 & 80.7 & 9 & 40.9 & 5 & 21.7 & 3 & 6.3 & $61.667 ; \mathrm{p}<0.01$ \\
\hline Q4 & 6 & 10.5 & 1 & 4.5 & 0 & 0.0 & 0 & 0.0 & $7.168 ; \quad p<0.01$ \\
\hline Q5 & 40 & 70.2 & 6 & 27.2 & 4 & 17.4 & 2 & 4.2 & $50.939 ; p<0.01$ \\
\hline Q6 & 19 & 33.3 & 1 & 4.5 & 1 & 4.3 & 0 & 0.0 & $23.837 ; p<0.01$ \\
\hline Q7 & 21 & 36.8 & 2 & 9.1 & 1 & 4.3 & 0 & 0.0 & $26.855 ; \mathrm{p}<0.01$ \\
\hline Q8 & 11 & 19.3 & 1 & 4.5 & 1 & 4.3 & 0 & 0.0 & $12.038 ; \mathrm{p}<0.01$ \\
\hline Q9 & 4 & 7.0 & 0 & 0.0 & 0 & 0.0 & 0 & 0.0 & $4.965 ; p<0.05$ \\
\hline
\end{tabular}

Table 3 shows the numbers and percentages of 'yes' response to each question in the scale by the four levels of WI. Percentages were calculated based on the number of households in each WI stratum (the response rate for each question in the scale was $100 \%)$. The response curves for each stratum of the WI were parallel to each other and the percentage of 'yes' responses (indicating food insecurity) to each question decreased with increasing level of wealth index, and vice versa (Figure 1). As the total number of 'yes' responses to question four and nine were less than others, caution in interpretation may be required. Linear trends observed across wealth index categories were statistically significant for all questions at $p<0.001$, except for the question nine which was significant at $\mathrm{p}<0.05$.

Similar to the findings related to WI, the response curves across HPCI were parallel to each other (except for question eight) and the percentage of 'yes' response for each question decreased with increasing level of HPCI, and vice versa. The linear trends across the levels 
Figure 1:'Yes' responses to questions in the HFIAS-SL by wealth index

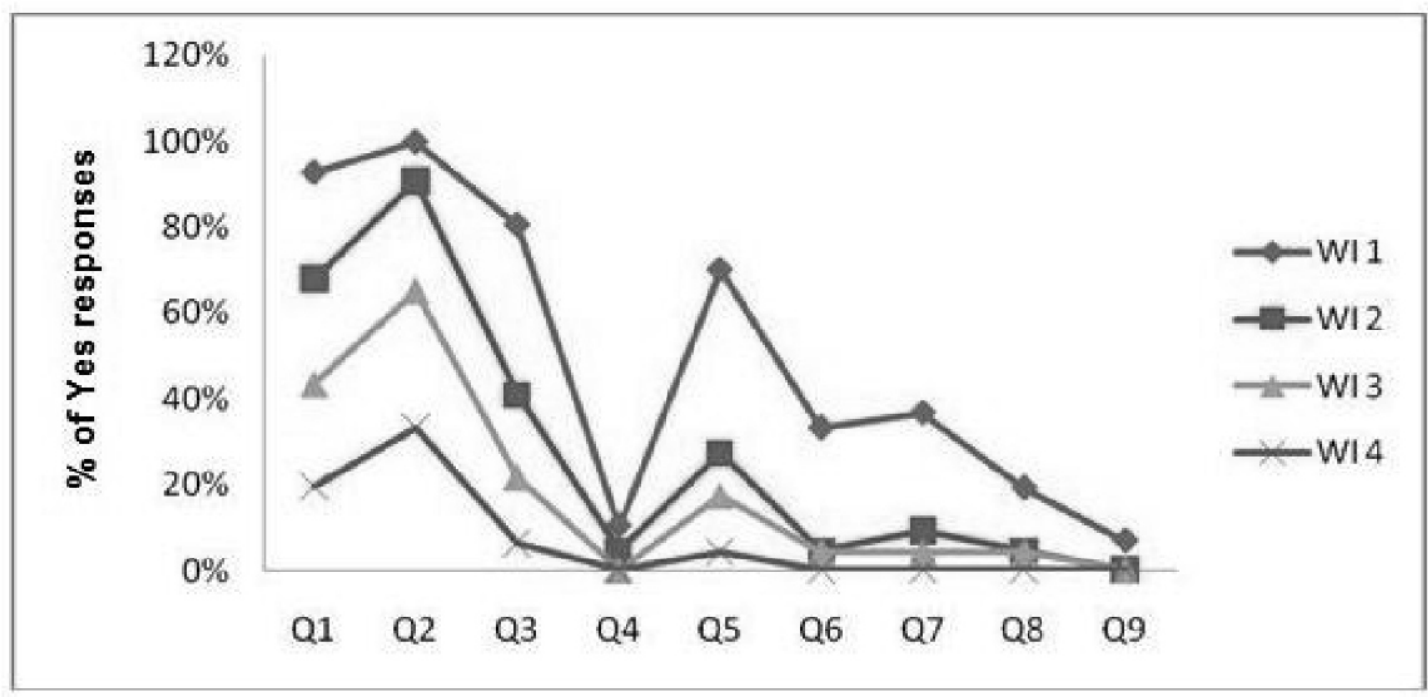

of the per capita income were statistically significant at $\mathrm{p}<0.01$ for all items, except question nine.

\section{Assessment of dose-response relationship} between level of household food insecurity and socio-economic strata

The proportion of households that were'food secure' and those that were 'severely food insecure' (the two extreme levels) were studied to examine its association with the two measures of the socio-economic strata of the households: WI and HPCI.None of the households belonging to the 'lowest WI' stratum was 'food secure' and none of the households belonging to the 'highest WI' stratum was 'severely food insecure' Considering

Figure 2: Percentage of 'food secure' and 'severely food insecure' households by wealth index

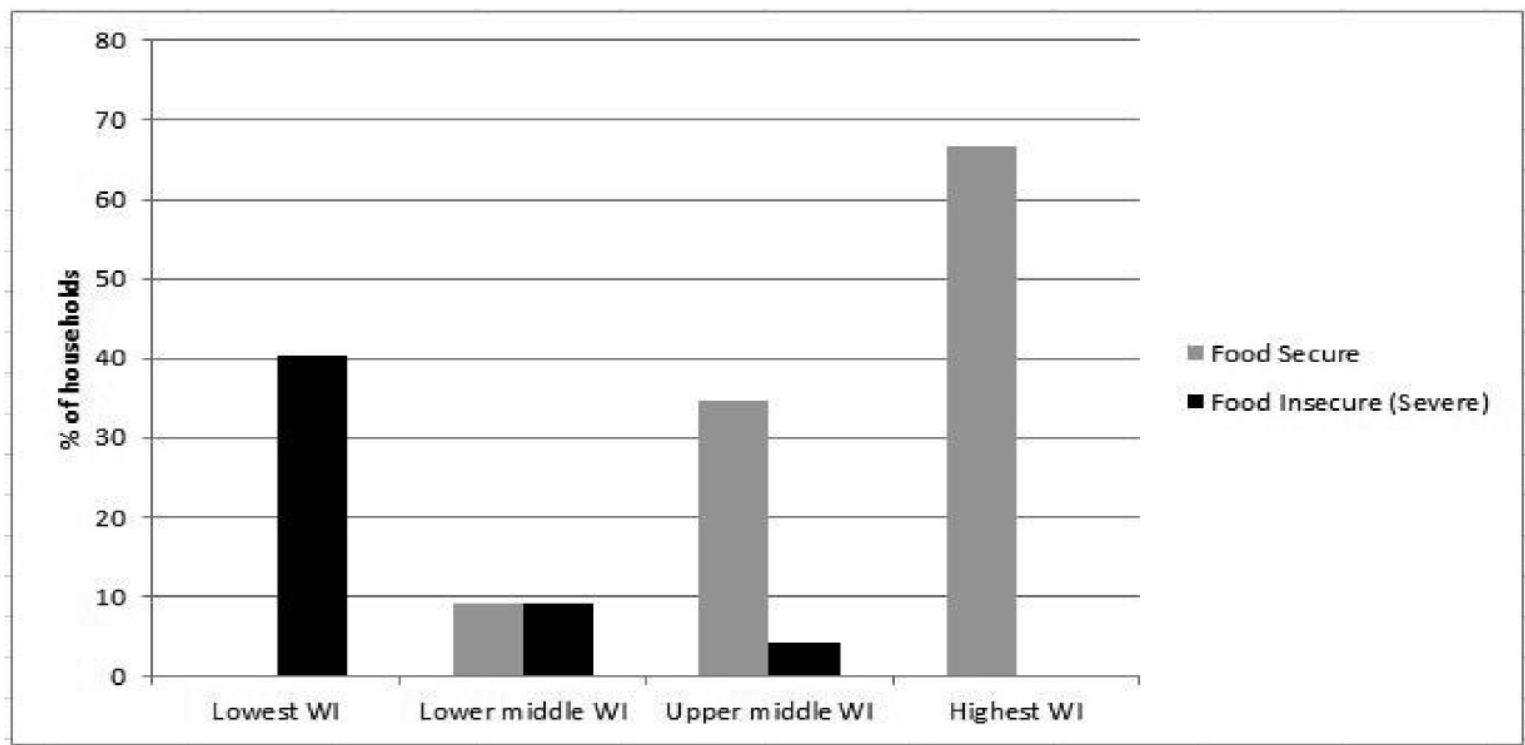

2. In Sri Lanka, foods from animal sources and fruits are the most expensive food items in general with almost all households consuming rice, vegetables and pulses. 
Table : 4 Household food insecurity by wealth index

\begin{tabular}{|c|c|c|c|c|c|c|c|c|c|}
\hline \multirow{3}{*}{$\begin{array}{l}\text { Household Food } \\
\text { Security/ } \\
\text { Insecurity }\end{array}$} & \multicolumn{8}{|c|}{ Wealth Index $(n=150)$} & \multirow{2}{*}{ Test for } \\
\hline & \multicolumn{2}{|c|}{ Lowest } & \multicolumn{2}{|c|}{ Lower } & \multicolumn{2}{|c|}{ Upper } & \multicolumn{2}{|c|}{ Highest } & \\
\hline & $(n=$ & & $\begin{array}{l}\text { mec } \\
(\mathrm{n}=\end{array}$ & & & $(n=23)$ & $(n=48)$ & & significance \\
\hline & No & $\%$ & No & $\%$ & No & $\%$ & No & $\%$ & $\begin{array}{l}\text { trend and } \mathrm{p} \\
\text { value) }\end{array}$ \\
\hline Food Secure & 0 & 0 & 2 & 9.1 & 8 & 34.8 & 32 & 66.7 & $\chi^{2}=43.484$ \\
\hline & & & & & & & & & $\mathrm{p}<0.001$ \\
\hline Mild Food & 9 & 15.8 & 12 & 54.5 & 10 & 43.5 & 14 & 29.2 & $\mathrm{p}>0.001$ \\
\hline Insecurity & & & & & & & & & \\
\hline Moderate Food & 25 & 43.9 & 6 & 27.3 & 4 & 17.4 & 2 & 4.2 & $\chi^{2}=36.086$ \\
\hline Insecurity & & & & & & & & & $\mathrm{p}<0.001$ \\
\hline Severe Food & 23 & 40.4 & 2 & 9.1 & 1 & 4.3 & 0 & 0 & $\chi^{2}=49.773$ \\
\hline Insecurity & & & & & & & & & $\mathrm{p}<0.001$ \\
\hline
\end{tabular}

the two extreme levels, 'food secure' and 'severely food insecure' categories, the data show a statistically significant linear trend $(p<0.001)$ across the wealth index strata in a dose-response manner. In this study, the trend was also linear and statistically significant for the 'moderate food insecure' category (Table 4). Similar to the findings on WI, the linear trends observed for the 'food secure' and 'severely food insecure' categories (two extreme levels) across HPCI strata were statistically significant $(\mathrm{p}<0.001)$. None of the households belonging to the 'lowest
HPCI' group was 'food secure' and none of the households belonging to the 'highest HPCI' group was 'severely food insecure'.

\section{Assessment of dose-response relationship between level of household food insecurity and consumption of 'selected food groups'}

To examine the relationship between HFI and the consumption of food within the household, those which consumed the selected food groups daily during the preceding week were identified and percentages of such households were assessed across the levels of household food insecurity. 


\section{Table : 5 Household with daily consumption of selected food items by the levels of household food insecurity}

\begin{tabular}{|c|c|c|c|c|c|c|c|c|c|}
\hline \multirow[t]{6}{*}{ Food groups } & \multicolumn{8}{|c|}{ Household Food Security/ Insecurity $(\mathrm{n}=150)$} & \multirow{6}{*}{$\begin{array}{l}\text { Test for } \\
\text { statistical } \\
\text { significance } \\
\text { ( } \chi^{2} \text { for linear } \\
\text { trend and } p \\
\text { value })\end{array}$} \\
\hline & \multicolumn{2}{|c|}{ Food secure } & \multicolumn{2}{|c|}{ Mild Food } & \multicolumn{2}{|c|}{ Moderate } & \multicolumn{2}{|c|}{ Severe } & \\
\hline & & & Ins & & Foo & & foo & & \\
\hline & & & & & Inse & & Inse & ure & \\
\hline & \multicolumn{2}{|c|}{$(n=42)$} & \multicolumn{2}{|c|}{$(\mathrm{n}=45)$} & \multicolumn{2}{|c|}{$(n=37)$} & \multicolumn{2}{|c|}{$(n=26)$} & \\
\hline & No & $\%$ & No & $\%$ & No & $\%$ & No & $\%$ & \\
\hline Food from & 28 & 66.7 & 18 & 40.0 & 8 & 21.6 & 3 & 11.5 & $\chi^{2}=33.653$ \\
\hline animal sources* & & & & & & & & & $\mathrm{p}<0.001$ \\
\hline Fruit & 22 & 52.4 & 14 & 31.1 & 3 & 8.1 & 2 & 7.7 & $\chi 2=32.587$ \\
\hline & & & & & & & & & $\mathrm{p}<0.001$ \\
\hline Milk & 34 & 81.0 & 29 & 64.4 & 30 & 81.1 & 8 & 30.8 & NS \\
\hline Rice & 37 & 88.1 & 30 & 66.7 & 37 & 100 & 22 & 84.6 & NS \\
\hline Vegetable & 37 & 88.1 & 30 & 66.7 & 35 & 94.6 & 22 & 84.6 & NS \\
\hline Pulses & 14 & 33.3 & 13 & 28.9 & 13 & 35.1 & 9 & 34.6 & NS \\
\hline
\end{tabular}

* meat/poultry/fish/eggs

The food groups included were: food from animal sources (meat/poultry/ fish/eggs), fruit, milk, rice, vegetables including green leaves and and pulses.

Table 5 shows that the daily consumption of foods from animal sources and fruit has a statistically significant dose-response relationship with the levels of HFI, in that the percentage of households that consumed these food items was highest in the 'food secure' group and lowest in the 'severely food insecure' group. Although milk did not show a dose response relationship, the percentage of households that consumed milk daily is significantly higher in the 'food secure' group than the 'severely food insecure' group $(\mathrm{p}<0.05)$. Daily consumption of other food groups (rice, vegetable and pulses) in the households did not show a relationship with the level of HFI. 


\section{Assessment of correlation between household food insecurity and socio-economic strata}

The correlation between the levels of HFI and socioeconomic strata represented by the wealth index and household per capita income was assessed using the Spearman correlation coefficient. This analysis showed that the correlation coefficient between HFI and WI is 0.759 and that between HFI and HPCI is 0.699. Both correlations were reasonably high and statistically significant at the level of $p<0.01$.

\section{Discussion}

The procedure used in the translation and adaptation of the HFIAS-SL to the Sri Lankan setting involved input and approval from both local content experts and target community members through which, face validity, consensual validity and content validity were appraised in the local setting. A similar procedure has been followed, where the USDA food insecurity scale was translated into local language and subsequently tested for content, consensual and face validity through content expert and focus groups made up of community members(3). As recommended by Frongillo (7), internal consistency, a measure to assess the internal validity of the instrument, measured by Cronbach's alpha must be 0.85 or higher to indicate adequate internal validity of the instrument(7). The internal validity of the HFIAS-SL was satisfactory, as the average measure of intra-class correlation coefficient (Cronbach's Alpha) is 0.879 ( $\mathrm{CI}=0.847-0.906)$, which fulfils the above criterion.

Assessment of external validity was carried out according to the approaches used and criteria recommended $(3,7)$. Therefore, it was necessary to have a sample of households purposively selected and belonging to different socioeconomic groups. This approach compares well with a study conducted in Brazil(3).
The presence of at least one child aged 12 to 59 months within a household was considered as an inclusion criterion because once validated, this tool can be applied in assessing HFI in households with children in this age group. Households which had resided in the study area for a period of less than one month were excluded as factors related to the initial settlement period could influence the food access and interfere with the relationships assessed during the validation procedure.

Several precautions were taken to improve the quality of data during the data collection. One author collected all HFI information while all other data was collected by one trained investigator. To improve the credibility of the validation procedure, a more objective measure, the wealth index, was included to represent the socio-economic status of the households in addition to the household per capita income.

This study found that the "Yes" response curves for each stratum of the WI were parallel to each other and the percentage of 'Yes' responses (indicating food insecurity) to each question decreased with increasing level of WI and vice versa. This is consistent with the findings from the study conducted in Brazil(3). The linear trends observed across wealth index categories were statistically significant for all items in the HFIAS-SL. Two extreme categories (food secure and severely food insecure households) were significantly associated in a dose-response manner with the WI and HPCI strata of the households, similar to the observation made by Perez-Escamilla et al(3). Food groups of animal origin and fruits were considered as nutritious and relatively expensive in the study area. Therefore they were assumed as food groups which were unlikely to be consumed 'daily' by the members of the food insecure households. It was also observed that the trends of daily consumption of foods from animal sources and 
fruits across the levels of the HFI show a statistically- significant dose-response relationship. Milk, though it is a food of animal origin, were considered as separate group because it is consumed by many people in daily basis irrespective of socio-economic status.

The findings of the validation procedure were consistent with Frongillo's proposed criteria for assessing the validity of food insecurity scales(7). In addition, the study showed a statistically significant correlation between the HFS and socio-economic strata of the household surveyed. Therefore, HFIAS-SL can be considered a valid tool for the assessment of household food insecurity in Sri Lankan settings.

\section{Acknowledgments:}

We are grateful to Dr. Renuka Jayathissa, Consultant Medical Nutritionist, Medical Research Institute, Dr Amal Harsha de Silva, Provincial Director, Western Province, Dr. Shanthi Gunawardhana Director, Nutrition Coordination Division and Mr. Sarath Gamage, Assistant Director (IT), Family Health Bureau, Ministry of Health for their assistance. The support extended by the Medical Officers and field staff in Kolonnawa and Pita-Kotte $\mathrm{MOH}$ areas is gratefully acknowledged. Our special thanks to Jenifer Coates, first author of HFIAS indicator guide (version 3) for her expert's opinion provided in relation to validation of HFIAS in this study. We are also grateful to the Education, Training and Research Division of the Ministry of Health for providing financial assistance.

\section{Disclosure of interest :No conflict of interest}

Funding : Education, Training and Research Division of the Ministry of Health, Sri Lanka

\section{References}

1. UNICEF. Unicef Policy Review; Strategy for improved nutrition of children and women in developing countries. New York, USA: UNICEF; 1990.

2. Coates J, Swindale A, Bilinsky P. Household Food Insecurity Access Scale (HFIAS) for Measurement of Household Food Access: Indicator Guide (v. 3), Washington, D.C.: Food and Nutrition Technical Assistance Project, Academy for Educational Development, August 2007.

3. Perez-Escamilla R, Segall-Corre AM, Maranha LK, Sampaio MFA, Marin-Leon L, Panigassi G. An adapted version of the U.S. Department of agriculture food insecurity module is a valid tool for assessing household food insecurity in Campinas, Brazil, $J$ Nutr.2004;134(8):1923-8.

4. Ministry of Health, UNICEF. Factors associated with complementary feeding in Sri Lanka; Sri Lanka complementary feeding study Colombo: Minstry of Health, Sri Lanka; 2008.

5. USAID. Policy Determination 19, Definition of Food Security. Washington DC: USAID; 1992.

6. Hamilton WL, Cook JT, Thompson WW, Buron LF, Frongillo EA, Olson CM, et al. Household food security in the United States in 1995: Summary report of the food security measurement project. Washington DC, USDA: 1997.

7. Frongillo E, A. Validation of measures of food insecurity and hunger. $J$ Nutr.1999; 129:506S9S. 
8. Abramson JH, Abramson ZH. Survey methods in community medicine: Epidemiological research and clinical trials. edition $5^{\text {th }}$, Edinburgh and New York: Churchill Livingstone; 1999.

9. Gonzalez W, Jimenez A, Madrigal G, Munoz LM, Frongillo EA. Development and Validation of Measure of Household Food Insecurity in Urban Costa Rica Confirms Proposed Generic Questionnaire.

J Nutr.2008;138:58792.
10. Frongillo EA, Nanama S, Wolfe WS. Development and validation of an experience based tool to directly measure household food insecurity within and across seasons in northern Burkina Faso, Washington, D.C.: 2004.

11. Department of Census and Statistics. Sri Lanka - Demographic and Health Survey 2006-2007. Colombo: Department of Census and Statistics, Sri Lanka 2009. 